PROCEEDINGS OF THE

AMERICAN MATHEMATICAL SOCIETY

Volume 126, Number 3, March 1998, Pages 645-646

S $0002-9939(98) 04063-5$

\title{
LIFTING UP AN INFINITE CHAIN OF PRIME IDEALS TO A VALUATION RING
}

\author{
BYUNG GYUN KANG AND DONG YEOL OH
}

(Communicated by Wolmer V. Vasconcelos)

\begin{abstract}
We prove that for an arbitrary chain $\left\{P_{\alpha}\right\}$ of prime ideals in an integral domain, there exists a valuation domain which has a chain of prime ideals $\left\{Q_{\alpha}\right\}$ lying over $\left\{P_{\alpha}\right\}$.
\end{abstract}

It is well-known that for a domain $D$ and a chain of prime ideals $P_{0} \subsetneq P_{1} \subsetneq$ .. $\subsetneq P_{l}$, there exists a valuation domain $V$ containing $D$ and a chain of prime ideals $Q_{0} \subsetneq Q_{1} \subsetneq \cdots \subsetneq Q_{l}$ lying over $P_{0} \subsetneq P_{1} \subsetneq \cdots \subsetneq P_{l}$ [3, Corollary 19.7]. This result is crucial in proving various results. The problem about a chain of prime ideals with an arbitrary length was posed by D. D. Anderson [1, \#7, p. 364]. In this paper, we answer this question affirmatively and prove that the above theorem [3, Corollary 19.7] is true for a chain of prime ideals of an arbitrary ordinal type. Throughout this paper, let $D$ be an integral domain with quotient field $K$.

Theorem. Let $D$ be an integral domain and let $\left\{P_{\alpha}\right\}$ be a chain of prime ideals in $D$. Then there exists a valuation overring $V$ of $D$ on $K$ with a chain of prime ideals $\left\{Q_{\alpha}\right\}$ such that $Q_{\alpha} \cap D=P_{\alpha}$.

Corollary. Let $\left\{P_{\alpha}\right\}$ be a chain of prime ideals in an integral domain $D$. Then there exists, in the integral closure of $D$, a chain of prime ideals $\left\{Q_{\alpha}\right\}$ lying over $\left\{P_{\alpha}\right\}$.

In order to prove the theorem, we need some lemmas.

Lemma 1. Let $\left\{P_{\alpha}\right\}_{\alpha \in \Lambda}$ be a chain of prime ideals of $D$. Let $S_{\alpha}=\left(D-P_{\alpha}\right)^{-1}$ $P_{\alpha}$ and $S=\bigcup_{\alpha} S_{\alpha}$. Then

(1) $S$ is closed under multiplication and $S$ is closed with respect to the multiplication by elements of $D$;

(2) $S_{\alpha}$ is closed under addition.

Proof. (1) Suppose that $a, b \in S$ and $c \in D$. Let $a=\frac{g}{f}, b=\frac{k}{h}$, where $a \in S_{\alpha}, b \in$ $S_{\beta}$. We may assume that $P_{\alpha} \subsetneq P_{\beta}$ by symmetry. Then $f h \notin P_{\alpha}$ since $f \notin P_{\alpha}$ and $h \notin P_{\beta}$. Since $g \in P_{\alpha}, g k \in P_{\alpha}$. Hence $a b \in S_{\alpha} \subseteq S$. Since $c g \in P_{\alpha}, c a \in S_{\alpha} \subseteq S$. Hence $S$ is closed under multiplication and with respect to the multiplication by elements of $D$.

(2) This follows because $S_{\alpha}$ is the maximal ideal of the ring $\left(D-P_{\alpha}\right)^{-1} D$.

Received by the editors May 16, 1996 and, in revised form, July 28, 1996.

1991 Mathematics Subject Classification. Primary 13A18; Secondary 13 B02.

This research was supported by the research grant BSRI-95-1431.

(C) 1998 American Mathematical Society 
Lemma 2. The ideal $\langle S\rangle$ generated by $S$ is a proper ideal in $D[S]$.

Proof. Let $P=\bigcup_{\alpha} P_{\alpha}$ and suppose $\langle S\rangle$ is not a proper ideal in $D[S]$. Then $1 \in\langle S\rangle$ in $D[S]$. By Lemma 1 ,

$$
1=s_{1}+\cdots+s_{n}, \quad \text { where } s_{k} \in S_{k} \text { for } k=1,2, \ldots, n .
$$

Let $s_{k}=\frac{g_{k}}{f_{k}}$, where $g_{k} \in P_{k}$ and $f_{k} \in D \backslash P_{k}$ - that is, $s_{k} \in S_{k}$ for each $k$. We may assume that $P_{n} \subsetneq P_{n-1} \subsetneq \cdots \subsetneq P_{2} \subsetneq P_{1}$. For the chain of prime ideals $P_{n} \subsetneq P_{n-1} \subsetneq \cdots \subsetneq P_{2} \subsetneq P_{1} \subsetneq P$, choose a valuation overring $V$ and a chain of prime ideals $Q_{n} \subsetneq Q_{n-1} \subsetneq \cdots \subsetneq Q_{2} \subsetneq Q_{1} \subsetneq Q$ lying over the given chain [3, Corollary 19.7]. For each $1 \leq i \leq n, g_{i} \in Q_{i}$ and $f_{i} \notin Q_{i}$ since $Q_{i} \cap D=P_{i}$ and $f_{i} \notin P_{i}$. Note that $\frac{f_{i}}{g_{i}} \notin V$ for otherwise $f_{i} \in g_{i} V \subseteq Q_{i}$. So $\frac{g_{i}}{f_{i}} \in V$. Therefore $g_{i} \in f_{i} V$, so $s_{i}=g_{i} / f_{i} \in V$. Since $s_{i} f_{i}=g_{i} \in Q_{i}$ and $f_{i} \notin Q_{i}$, it follows that $s_{i} \in Q_{i}$. This implies that $1=\frac{g_{1}}{f_{1}}+\cdots+\frac{g_{n}}{f_{n}} \in Q$ contrary to the fact that $Q$ is a proper ideal. Hence $\langle S\rangle$ is a proper ideal in $D[S]$.

Proof of the main theorem. Since $D_{P} \cap D=D$ and $P D_{P} \cap D=P[2$, Theorem 34], we may assume that $P$ is a maximal ideal of $D$. Let $Q$ be a prime ideal containing $\langle S\rangle$ in $D[S]$. Then there exists a valuation overring $V$ of $D[S]$ such that $M \cap D[S]=Q$, where $M$ is the maximal ideal of $V[3$, Theorem 19.6]. Since $P$ is a maximal ideal in $D, M \cap D=P$. We have only to show that $\sqrt{P_{\alpha} V} \cap D=P_{\alpha}$. Suppose that it fails. We choose an element $f \in \sqrt{P_{\alpha} V} \cap D \backslash P_{\alpha}$. Then $f \in D \backslash P_{\alpha}$ and $f^{n} \in P_{\alpha} V, n \in \mathbb{N}$. So $\frac{f^{n}}{g} \in V, g \in P_{\alpha}$. This implies $\frac{g}{f^{n}} \in S$ is a unit in $V$. This contradicts the fact that $\langle S\rangle \subseteq M$. Hence $\sqrt{P_{\alpha} V} \cap D=P_{\alpha}$. Furthermore, $\sqrt{P_{\alpha} V}$ is a prime ideal in $V[3$, Theorem 17.1].

\section{REFERENCES}

[1] D. D. Anderson, Some Problems in Commutative Ring Theory, Zero-Dimensional Commutative Rings (Lecture Notes in Pure and Applied Mathematics Series/171 edited by D. F. Anderson and D. E. Dobbs), Marcel Decker, New York, 1995. MR 96a:13001

[2] I. Kaplansky, Commutative Rings, The University of Chicago Press, 1974. MR 49:10674

[3] R. Gilmer, Multiplicative Ideal Theory, Marcel Decker, New York, 1972. MR 55:323; MR 93j: 13001

Department of Mathematics, Pohang University of Science \& Technology, Pohang, 790-784, KOREA

E-mail address: bgkang@euclid.postech.ac.kr 